Research Paper

\title{
Expression of Notch Gene and Its Impact on Survival of Patients with Resectable Non-small Cell Lung Cancer
}

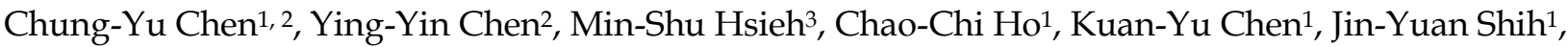 \\ Chong-Jen $\mathrm{Yu}^{1 凶}$ \\ 1. Division of Pulmonary and Critical Care Medicine, Department of Internal Medicine, National Taiwan University Hospital, College of Medicine, National \\ Taiwan University, Taipei, Taiwan; \\ 2. Department of Internal Medicine, National Taiwan University Hospital Yunlin Branch, Yunlin County, Taiwan; \\ 3. Department of Pathology and Graduate Institute of Pathology, National Taiwan University Hospital, College of Medicine, National Taiwan University, \\ Taipei, Taiwan. \\ $\square$ Corresponding author: Chong-Jen Yu, MD, PhD, Division of Pulmonary and Critical Care Medicine, Department of Internal Medicine, National Taiwan \\ University Hospital, No.7, Chung-Shan South Road, Taipei 100, Taiwan Tel: 886-2-2356-2905; Fax: 886-2-2358-2867 Email: jefferycjyu@ntu.edu.tw \\ ( $)$ Ivyspring International Publisher. This is an open access article distributed under the terms of the Creative Commons Attribution (CC BY-NC) license \\ (https://creativecommons.org/licenses/by-nc/4.0/). See http://ivyspring.com/terms for full terms and conditions.
}

Received: 2016.09.28; Accepted: 2017.02.08; Published: 2017.05.11

\begin{abstract}
BACKGROUND: Notch signaling has been demonstrated to frequently participate in the process of lung carcinogenesis. This study aimed to search Notch expression in non-small cell lung cancer (NSCLC) and its impact on survival.

METHODS: From 2001 to 2011 , patients with diagnosis of NSCLC who received surgical resection were included. The expression of Notch gene was assessed by real-time polymerase chain reaction (RT-PCR). Clinical characteristics, histological types, disease stages, and outcomes were analyzed.

RESULTS: Ninety-seven patients with NSCLC being explored the expression of Notch gene (Notch 1 - 4). Seventy-five patients $(77.3 \%)$ were adenocarcinoma. Patients with adenocarcinoma had higher expression of Notch2 than other histology types $(p<0.001)$. Otherwise, patients with squamous cell carcinoma had relative higher expression of Notch 1 and Notch 3 expression $(p=$ 0.014 and $p=0.032$, respectively). Notch2 expression increased associated with patients with more advanced lung cancer stage. Patients who had cancer recurrence also had higher Notch2 expression $(p=0.008)$. The patient group with lung adenocarcinoma of both high Notch 1 and Notch3 expression had a shorter median disease-free survival (DFS) (both high v.s both low: DFS, median, 7.2 v.s 25.3 months, $p=0.03$ ). However, the expression of Notch gene had no impact on overall survival.

CONCLUSIONS: Patients with lung adenocarcinoma had higher Notch2 expression. Patients with higher Notch2 expression also had higher rate of cancer recurrence. Both higher Notchl and Notch 3 expression was associated with poor prognosis in lung adenocarcinoma.
\end{abstract}

Key words: NOTCH, lung cancer, carcinogenesis, biomarker, prognosis.

\section{Introduction}

Lung cancer is a leading cause of cancer death in many countries, and non-small cell lung cancer (NSCLC) accounts for around $85 \%$ of lung cancers (1). Despite the advance of different therapeutic regimens, the 5-year survival of NSCLC has remained at $15 \%$ in the past decades (2). It is therefore imperative to deepen the understanding of the molecular mechanism of NSCLC, and to find novel therapeutic strategies for this disease.

Notch signaling has been demonstrated to frequently participate in the process of lung development and lung carcinogenesis (3-8). Notch signaling pathway is associated with angiogenesis which is an essential step for tumor growth and 
progression and plays a vital role in tumor invasion and metastasis (9-11). The mammalian Notch family consists of four receptors, Notch1-4, and five ligands, Jagged 1 and 2, and Delta-like ligand1, 3, and 4 (DLL1, $-3,-4)$. The majority of studies consider that Notch1 is involved in cancer promotion $(12,13)$. It is hypothesized that Notch1 and 3 are associated with $\operatorname{NSCLC}(14,15)$.

Several studies demonstrated that Notch expression is distinct in different histology type of lung cancer (16-19). Though, the diverse function between Notch1 to Notch4 is not well known. The activation status of Notch had poor prognostic impact on lung cancer patients' survival (20). Recent work in Notch pathway activity in cancer cells shows epithelial -to- mesenchymal transition (EMT) promotion (21-24), enhancement of cancer stem cell characteristics (25), and displaying resistance to conventional chemotherapy $(26,27)$. Higher activities of Notch gene in lung cancer indicated worse survival (28).

Until now, the role of Notch in lung cancer development remains elusive. Otherwise, the association of expression of Notch gene with its impact on prognosis in NSCLC has not be well clarified. Here, we will describe the expression of Notch1 to Notch4 gene in different NSCLC histology types and investigate the influence of Notch expression on survival.

\section{Material and Methods}

\section{Patient data collection}

From the year 2001 to 2011, patients with diagnosis of NSCLC who received any surgical resection as lobectomy or wedge resection at National Taiwan University Hospital were included.

Disease stages were reviewed and determined by the 7th version of the TNM staging system for lung cancer of the International Association for the Study of Lung Cancer (IASLC). The medical records were analyzed for age, gender, smoking history, underlying diseases, histology type, tumor staging, recurrent or metastatic site.

Information on survival was obtained through active follow-up based on the verification of patients' vital status. The disease-free survival (DFS) was defined as the time from the surgical intervention to disease progression or death from any cause. It was determined as the date of the last follow-up visit for patients who were still alive and who had no disease progression. The overall survival (OS) was defined as the time from the date of beginning treatment to the date of death or last follow-up by August 2013.

\section{Notch gene expression analysis}

The expression of Notch elements was assessed by quantitation real-time polymerase chain reaction (RT-PCR). Taqman Gene expression assay with TaqMan ${ }^{\circledR}$ RNA-to-Ct ${ }^{\mathrm{TM}}$ 1-Step Kit was used to analysis Notch1 - 4 from cancer tissues. Standard curve with gene expression amplification was plotted to difference in gene expression and standard deviation in the replicate groups. Ct values for each well and for each replicate group was calculated.

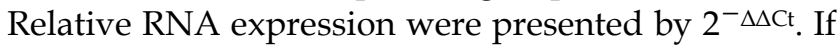
$2^{-} \Delta \Delta \mathrm{Ct}>1$ is defined as higher Notch expression.

\section{Immunohistochemistry (IHC) stain of Notch2}

Formalin fixed paraffin-embedded sections were deparaffinized and rehydrated (Xylene 1:1 with $100 \%$ ethanol, $100 \%$ ethanol down to $50 \%$ ethanol). Next, the sections were heated by microwave in citrate buffer pH6.5 for $20 \mathrm{~min}$ total for antigen retrieval. Then block endogenous peroxidases in 90 $\mathrm{ml}$ methanol $/ 10 \mathrm{ml} \mathrm{30 \%} \mathrm{H} 2 \mathrm{O} 2$ for 10 minutes at room temperature was performed, followed by wash in PBS. Slides were then incubated with primary antibodies for Notch2 (ab8926, Abcam, diluted 1:100), overnight at $4 \circ \mathrm{C}$. The immunohistochemical reaction was visualized and analyzed using a microscope. Peripheral normal lung tissue was used for the negative controls. Immunostaining was defined independently by two pathologists blinded to the clinical data and scored by multiplying the intensity of staining and the percentage of the stained tumor cells. The intensity of staining was graded as follows: 0 (colorless), 1 (weak, pallide-flavens), 2 (moderate, yellow), and3 (strong, brown).

Tumors with weak, moderate or strong immunostaining intensity were classified as positive $(+)$ expression, whereas tumors with absent immunostaining were classified as negative expression.

\section{Statistical Analysis}

Continuation variables are expressed as mean with standard deviation and categorical variables are expressed as a percentage of the group from which they were derived. The proportion between groups were compared by Pearson chi-square test or Fisher exact test. ANOVA analysis with Post Hoc was used to difference the expression of Notch1 $-4\left(2^{-\Delta \Delta C t}\right)$ in different histology type. Cox regression model was used for covariate analysis to determine the hazard ratio of Notch expression and survival. A $p$ value less than 0.05 was considered significant. All analyses were performed using the SPSS software (version 16, SPSS Institute, Chicago, IL). 


\section{Results}

\section{Patients' clinical characteristics and the association of Notch gene expression}

From 2001 to 2011, ninety-seven patients with NSCLC received surgical intervention being explored the expression of Notch gene (Notch1 - 4). Fifty-eight patients $(59.8 \%)$ were male. The mean age was $67.0 \pm$ 11.1 years old. Seventy-five patients $(77.3 \%)$ were adenocarcinoma, 19 patients $(19.6 \%)$ were squamous cell carcinoma. Most patients $(n=52,53.6 \%)$ were stage I disease (Table 1).

Female patients had higher Notch2 expression (female v.s. male, $2^{-\Delta \Delta C t}$, mean $\pm \mathrm{SD}$, Notch2: $1.21 \pm$ 0.38 v.s. $1.01 \pm 0.49, p=0.03$ ). Patients who were never smoking also had higher Notch2 expression (never smoking v.s. current or ever smoking, $2-\Delta \Delta \mathrm{Ct}$, mean \pm SD, Notch 2: $1.21 \pm 0.43$ v.s. $0.98 \pm 0.46, p=0.02$ ). Nevertheless, patients who were never smoker had lower Notch3 and Notch4 expression (never smoker v.s. current or ever smoker, $2^{-\Delta \Delta \mathrm{Ct}}$, mean $\pm \mathrm{SD}$, Notch3: $1.52 \pm 0.98$ v.s. $3.09 \pm 4.61, p=0.02$; Notch4: $0.32 \pm 0.19$ v.s. $0.42 \pm 0.28, p=0.04$, respectively) (Table 1 ).

\section{Notch expression in different histology type}

Patients with adenocarcinoma had higher expression of Notch2 than other histology types (adenocarcinoma, v.s. squamous cell carcinoma, v.s others, $2-\Delta \Delta \mathrm{Ct}$, mean $\pm \mathrm{SD}$, Notch2: $1.23 \pm 0.42$ v.s. $0.64 \pm$ 0.20 , v.s. $0.54 \pm 0.29, p<0.001)$. Otherwise, patients with squamous cell carcinoma had relative higher expression of Notch1 and Notch3 expression (adenocarcinoma, v.s. squamous cell carcinoma, v.s others, $2-\Delta \Delta C t$, mean $\pm S D$, Notch1: $0.73 \pm 0.36$ v.s. $1.02 \pm$ 0.47 , v.s. $1.01 \pm 0.73, p=0.01$; and Notch3: $1.86 \pm 1.48$ v.s. $4.17 \pm 7.06$, v.s. $2.30 \pm 1.75, p=0.03$, respectively) (Table 1 and Figure 1).

\section{Notch expression in lung cancer stage}

Notch2 expression increased associated with patients with more advanced lung cancer stage (linear regression analysis between Notch2 expression and lung cancer stage: $\mathrm{r}$ square $=0.094, p$ value $=0.002$ ). However, expression of Notch1, Notch3 and Notch4 had no correlation with lung cancer stage in statistic (Figure 2A). Expression of Notch2 was significant higher in patients with stage IIIA disease compared to patients with stage I (stage IIIA v.s. stage I, 2- $\Delta \Delta \mathrm{Ct}$, mean \pm SD, Notch2: $1.41 \pm 0.54$ v.s. $0.96 \pm 0.37, p$ value $<0.001$ ) (Figure 2B).

\section{Immunohistochemistry stain of Notch2 in lung adenocarcinoma}

Notch2 expression in 16 of 75 (21.3\%) lung adenocarcinoma was also detected by immunohistochemistry (IHC) stain to confirm the reliance of Notch2 expression level calculated by RT-PCR. Figure 3(A) and 3(B) negative Notch2 expression (RT-PCR $2-\Delta \Delta$ Ct 0.60 to 0.77 ). Figure $3(\mathrm{C})$ and 3(D) showed weak Notch2 expression (faint) (RT-PCR 2- $\Delta \Delta$ Ct 0.82 to 0.98). Figure 3(E) and 3(F) showed moderate Notch2 expression (RT-PCR 2$\Delta \Delta \mathrm{Ct} 1.25$ to 1.34$)$. Notch2 was scarcely stained (yellow) in the cytoplasm of tumor cells. Figure 3(G) and $3(\mathrm{H})$ showed high Notch2 expression (brown) in adenocarcinoma (RT-PCR $2-\Delta \Delta$ Ct 2.30 to 2.72). Notch2 was strongly stained in the cytoplasm of tumor cells. The score of Notch2 IHC stain was consistent with the result of RT-PCR.

Table 1. Demography data of 97 patients with NSCLC for Notch gene analysis

\begin{tabular}{|c|c|c|c|c|c|c|c|c|c|}
\hline Patient characteristics & $\mathrm{N}(\%)$ & $\begin{array}{l}\text { Notch1 } \\
(\text { mean } \pm \text { SD) }\end{array}$ & $p$ value & $\begin{array}{l}\text { Notch2 } \\
(\text { mean } \pm S D)\end{array}$ & $p$ value & $\begin{array}{l}\text { Notch3 } \\
(\text { mean } \pm S D)\end{array}$ & $p$ value & $\begin{array}{l}\text { Notch4 } \\
(\text { mean } \pm S D)\end{array}$ & $p$ value \\
\hline \multicolumn{10}{|l|}{ Age, years old } \\
\hline Age $<70$ & $48(49.5 \%)$ & $0.84 \pm 0.36$ & \multirow[t]{2}{*}{0.29} & $1.07 \pm 0.50$ & \multirow[t]{2}{*}{0.62} & $2.83 \pm 4.71$ & \multirow[t]{2}{*}{0.16} & $0.39 \pm 0.27$ & \multirow[t]{2}{*}{0.50} \\
\hline Age $\geq 70$ & $49(50.5 \%)$ & $0.75 \pm 0.45$ & & $1.12 \pm 0.42$ & & $1.83 \pm 1.29$ & & $0.36 \pm 0.23$ & \\
\hline \multicolumn{10}{|l|}{ Gender } \\
\hline Male & $58(59.8 \%)$ & $0.83 \pm 0.43$ & \multirow[t]{2}{*}{0.33} & $1.01 \pm 0.49$ & \multirow[t]{2}{*}{0.03} & $2.89 \pm 4.34$ & \multirow[t]{2}{*}{0.05} & $0.40 \pm 0.28$ & \multirow[t]{2}{*}{0.16} \\
\hline Female & $39(40.2 \%)$ & $0.74 \pm 0.36$ & & $1.21 \pm 0.38$ & & $1.50 \pm 0.86$ & & $0.33 \pm 0.18$ & \\
\hline \multicolumn{10}{|l|}{ Smoking status } \\
\hline Never & $47(48.5 \%)$ & $0.77 \pm 0.41$ & \multirow[t]{2}{*}{0.50} & $1.21 \pm 0.43$ & \multirow[t]{2}{*}{0.02} & $1.52 \pm 0.98$ & \multirow[t]{2}{*}{0.02} & $0.32 \pm 0.19$ & \multirow[t]{2}{*}{0.04} \\
\hline Ever or current smoking & $50(51.5 \%)$ & $0.82 \pm 0.40$ & & $0.98 \pm 0.46$ & & $3.09 \pm 4.61$ & & $0.42 \pm 0.28$ & \\
\hline \multicolumn{10}{|l|}{ Histology } \\
\hline Adenocarcinoma & $75(77.3 \%)$ & $0.73 \pm 0.36$ & \multirow[t]{2}{*}{0.01} & $1.23 \pm 0.42$ & \multirow[t]{2}{*}{$<0.001$} & $1.86 \pm 1.48$ & \multirow[t]{2}{*}{0.03} & $0.34 \pm 0.20$ & \multirow[t]{2}{*}{0.06} \\
\hline $\begin{array}{l}\text { Squamous cell } \\
\text { carcinoma }\end{array}$ & $19(19.6 \%)$ & $1.02 \pm 0.47$ & & $0.64 \pm 0.20$ & & $4.17 \pm 7.06$ & & $0.48 \pm 0.36$ & \\
\hline Others & $3(3.1 \%)$ & $1.01 \pm 0.73$ & & $0.54 \pm 0.29$ & & $2.30 \pm 1.75$ & & $0.48 \pm 0.27$ & \\
\hline \multicolumn{10}{|l|}{ Disease stage } \\
\hline I & $52(53.6 \%)$ & $0.76 \pm 0.41$ & \multirow[t]{3}{*}{0.45} & $0.96 \pm 0.37$ & \multirow[t]{3}{*}{0.002} & $2.64 \pm 4.58$ & \multirow[t]{3}{*}{0.80} & $0.37 \pm 0.29$ & \multirow[t]{3}{*}{0.81} \\
\hline II & $25(25.8 \%)$ & $0.80 \pm 0.37$ & & $1.15 \pm 0.44$ & & $1.97 \pm 1.18$ & & $0.36 \pm 0.18$ & \\
\hline IIIA & $18(18.6 \%)$ & $0.91 \pm 0.44$ & & $1.41 \pm 0.54$ & & $2.06 \pm 1.35$ & & $0.43 \pm 0.21$ & \\
\hline \multicolumn{10}{|l|}{ Cancer recurrence } \\
\hline Yes & $45(46.4 \%)$ & $0.84 \pm 0.42$ & \multirow[t]{2}{*}{0.36} & $1.22 \pm 0.51$ & \multirow[t]{2}{*}{0.008} & $2.14 \pm 1.77$ & \multirow[t]{2}{*}{0.63} & $0.38 \pm 0.23$ & \multirow[t]{2}{*}{0.81} \\
\hline No & $52(53.6 \%)$ & $0.76 \pm 0.40$ & & $0.98 \pm 0.37$ & & $2.49 \pm 4.44$ & & $0.37 \pm 0.26$ & \\
\hline
\end{tabular}



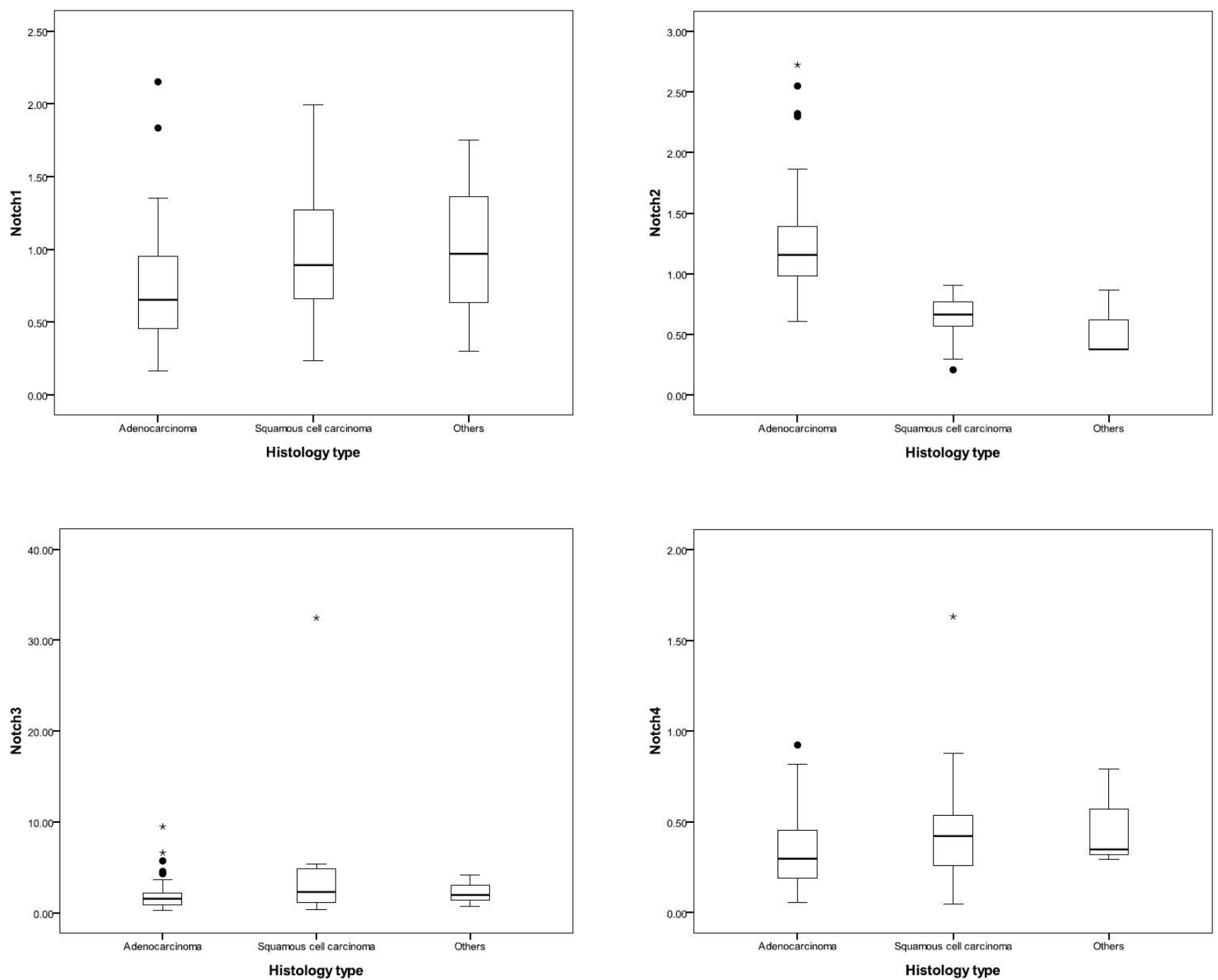

Figure 1. Notch expression in different histological types. The line of box indicated the median of Notch expression, and the box presented the 25th to 75 th percentile of Notch expression. Patients with adenocarcinoma had a higher expression of Notch2, and patients with squamous cell carcinoma had a relatively higher expression of Notch1 and Notch3.

\section{The effect of Notch expression on survival}

Patients with adenocarcinoma had significantly higher recurrence rate $(39 / 75,52.0 \%)$ than those with squamous cell carcinoma $(5 / 19,26.3 \%)(p=0.045)$. Patients who had cancer recurrence also had higher Notch2 expression (cancer recurrence v.s none, $2-\Delta \Delta \mathrm{Ct}$, mean \pm SD, $1.22 \pm 0.51$, v.s. $0.98 \pm 0.37, p=0.008$ ).

Patients with adenocarcinoma who had higher Notch1 or Notch3 expression had poor disease-free survival (DFS) (DFS, median, lower Notch1 v.s. higher Notch1, 21.2 v.s 7.2 months, $\mathrm{p}=0.03$; and lower Notch 3 v.s. higher Notch3, 25.3 v.s. 12.0 months, $p=$ 0.05 , respectively). (Figure $4 \mathrm{~A}$ and $4 \mathrm{~B}$ ). The expression of Notch signaling had no impact on overall survival (OS). Otherwise, expression of Notch signaling had no impact on DFS and OS in other histology types.
We investigated the association of combined Notch 1 and Notch 3 expressions and survival. There was also a shorter median DFS in the patient group of both high expression $(n=14)$ of Notch1 and Notch3 than those with both low expression of Notch1 and Notch3 group $(n=23)$ (both high vs. others v.s both low: DFS, median, 7.2 v.s. 14.2 v.s 25.3 months, $p=$ 0.03) (Figure 4C).

Cox regression model was used to analysis the expression of Notch1 to Notch4 associated with DFS in lung adenocarcinoma. The expression of Notch3 remained an important predictive factor of DFS. Lung adenocarcinoma patients with higher expression of Notch3 had higher risk of disease progression, however, this impact on PFS was not significant statistically (hazard ratio 1.20; 95\% confidence interval, 0.99 to $1.46, p=0.06$ ) (Table 2). 

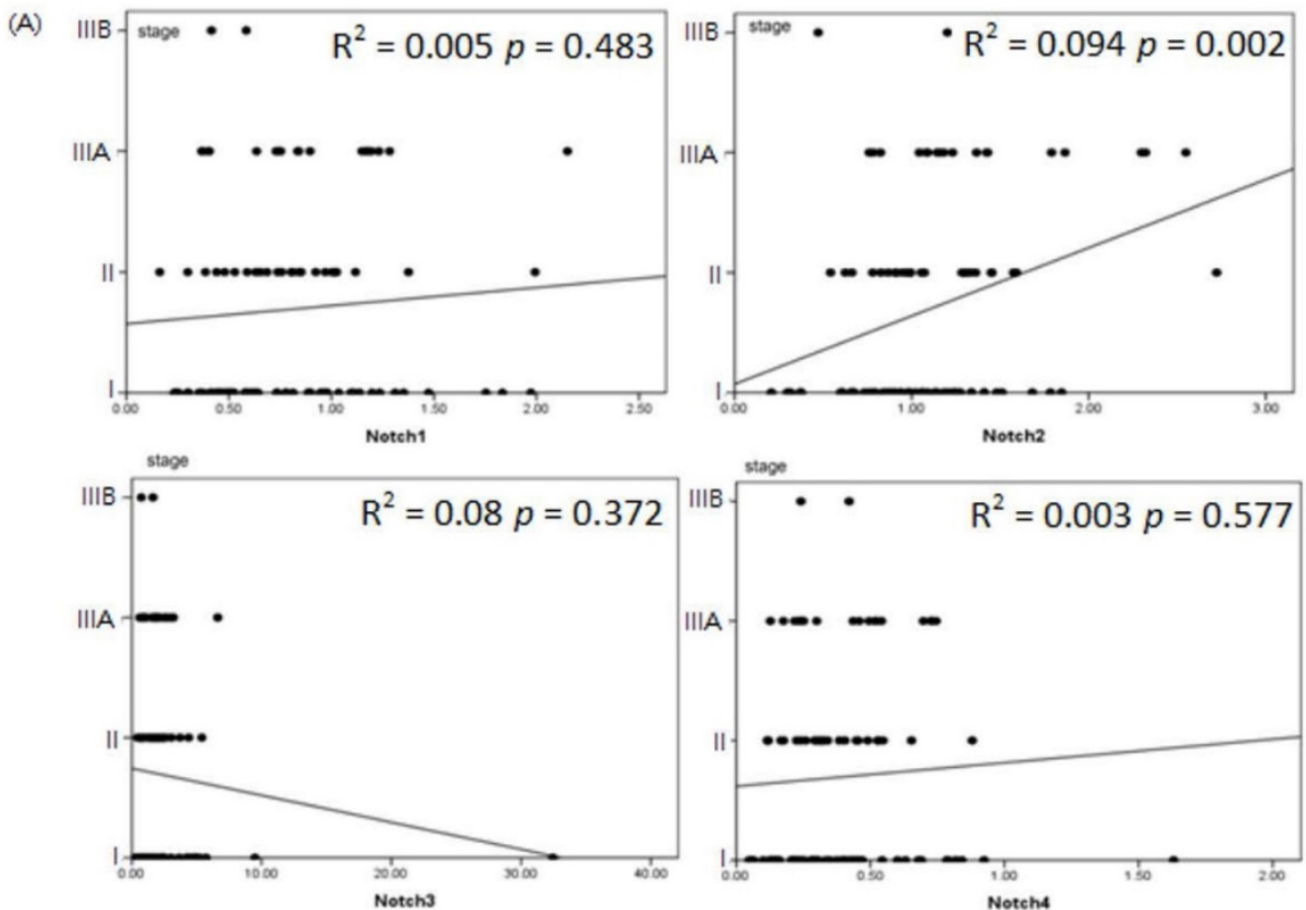

(B)

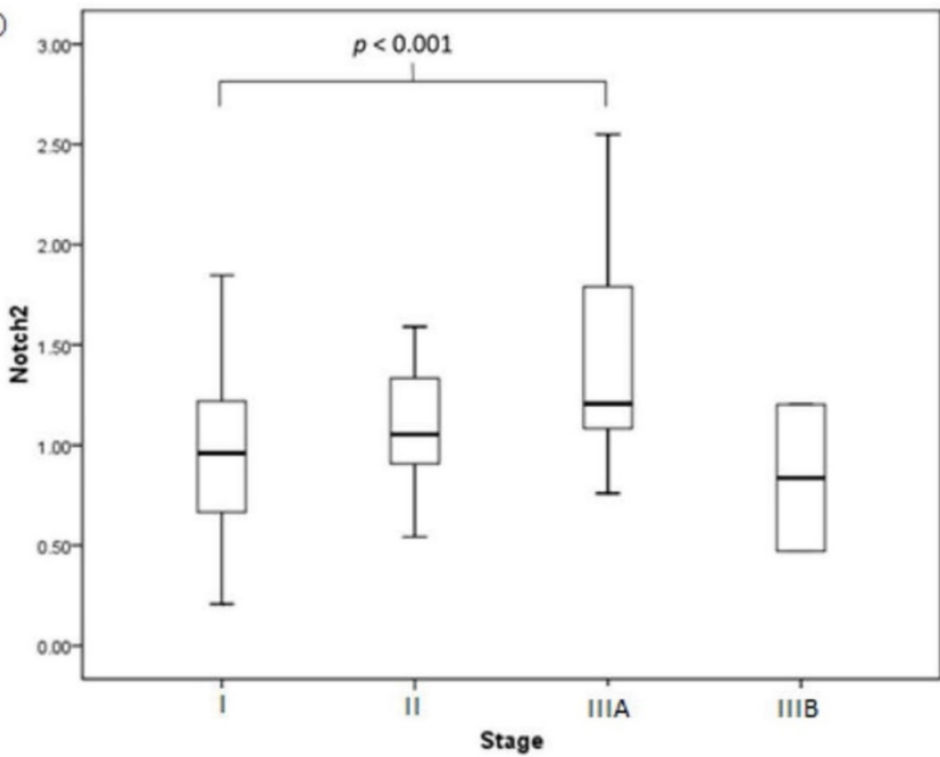

Figure 2. Notch expression associate with lung cancer stage. (A) The association of Notch1 to Notch4 and lung cancer stage was analyzed by linear regression. Notch2 expression increased associated with patients with more advanced lung cancer stage $(r$ square $=0.094, p$ value $=0.002)$. (B) Expression of Notch2 was significant higher in patients with stage IIIA disease compared to patients with stage I ( $p$ value $<0.001)$.

Table 2. Cox regression model to analysis Notch gene expression associated with disease-free survival (DFS) in patients with lung adenocarcinoma

\begin{tabular}{lllll}
\hline & P value & HR & & $95 \% \mathrm{CI}$ \\
\cline { 4 - 5 } & & & Lower & Upper \\
\hline Notch1 & 0.11 & 2.05 & 0.85 & 4.97 \\
Notch2 & 0.66 & 1.17 & 0.57 & 2.41 \\
Notch3 & 0.06 & 1.20 & 0.99 & 1.46 \\
Notch4 & 0.84 & 0.84 & 0.16 & 4.37 \\
\hline
\end{tabular}

HR: Hazard ration; CI: Confidence interval

\section{Discussion}

In the present study, we examined the expression of Notch1 to Notch4 signaling expression in resected NSCLC tissues by RT-PCR, compare with the adjacent normal lung tissues from the same patient. Patients who were female or never smoking had higher Notch2 expression. Patients with lung adenocarcinoma had higher expression of Notch2 than other histology types. On the other hand, 
patients with squamous cell carcinoma had relative higher expression of Notch1 and Notch3 expression. The association of Notch2 expression in NSCLC correlated with cancer recurrence or metastasis. However, higher Notch 1 and Notch 3 expression may be related to disease progression of lung adenocarcinoma.

Recent study demonstrated that Notch1 expression is higher in lung squamous cell carcinoma (16) and Notch 3 is over-expressed in about $40 \%$ of
NSCLC (17-19). In our study, patients with lung cancer who had smoking history had higher Notch3 expression. Notch1 and Notch3 expression were consistently higher in lung squamous cell carcinoma. Besides, we also find that patients who were female, never smoking and with lung adenocarcinoma, have significant higher Notch2 expression. Therefore, this finding approves that Notch2 signaling plays an important role in mutation of lung adenocarcinoma (20), especially in never-smoking female at east Asia.
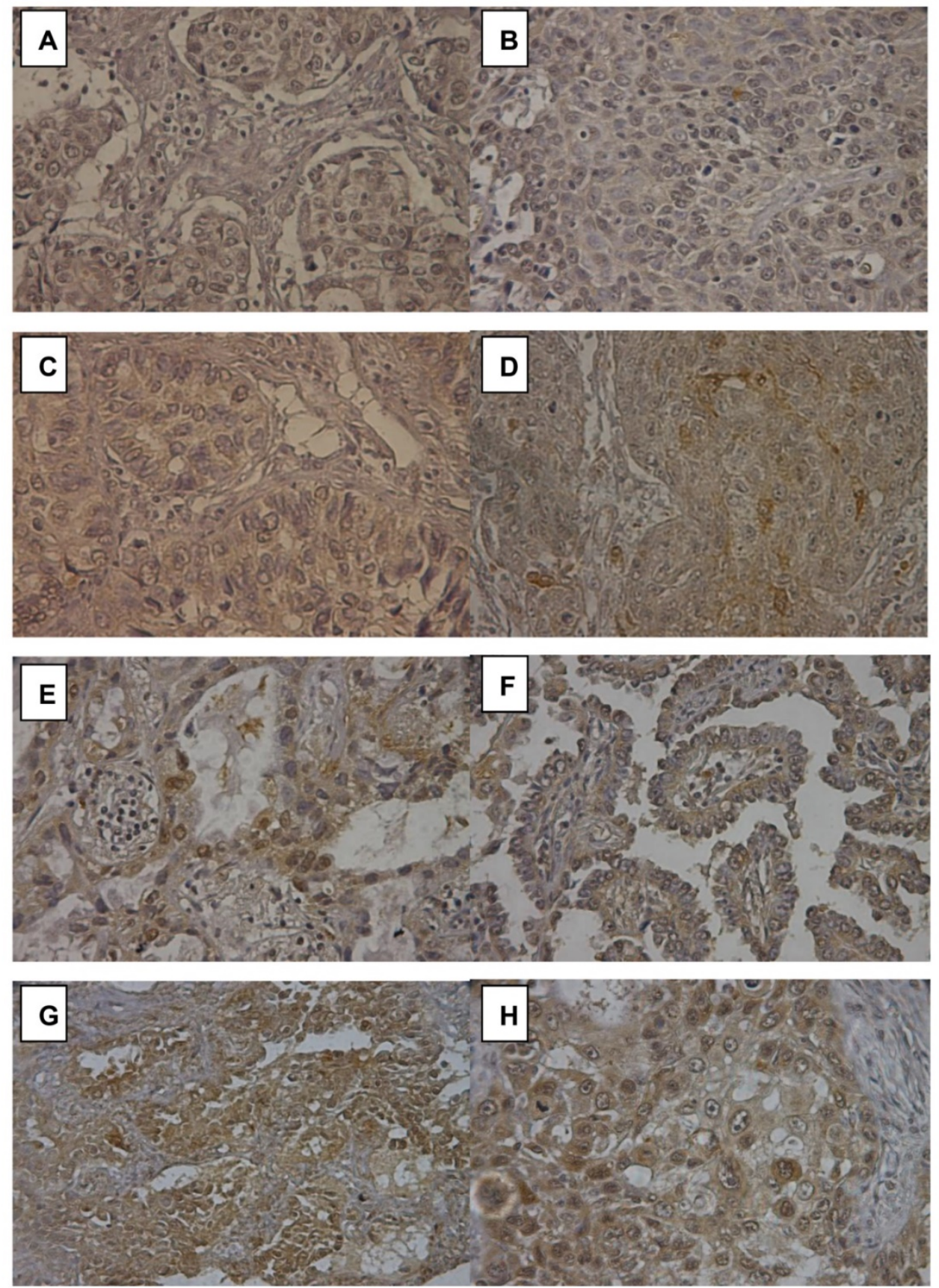

Figure 3. Immunohistochemistry (IHC) stain of Notch2 in lung adenocarcinoma. (A) and (B) showed negative Notch2 expression. (C) and (D) showed weak Notch2 expression (faint). $(E)$ and $(F)$ showed moderate Notch2 expression. Notch2 was scarcely stained (yellow) in the cytoplasm of tumor cells. (G) and (H) showed high Notch 2 expression (brown) in adenocarcinoma. Notch2 was strongly stained in the cytoplasm of tumor cells. Peripheral normal tissue was a negative control in the same section. 
(A)

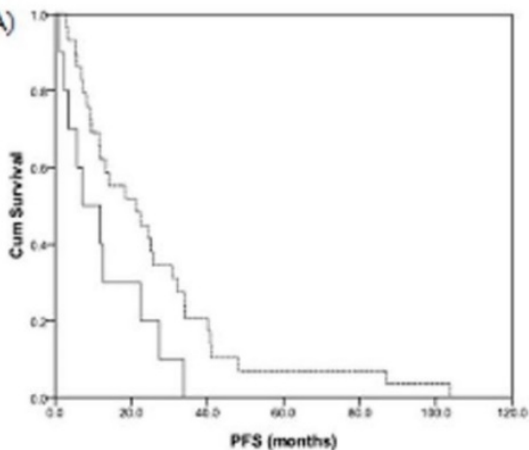

(B)

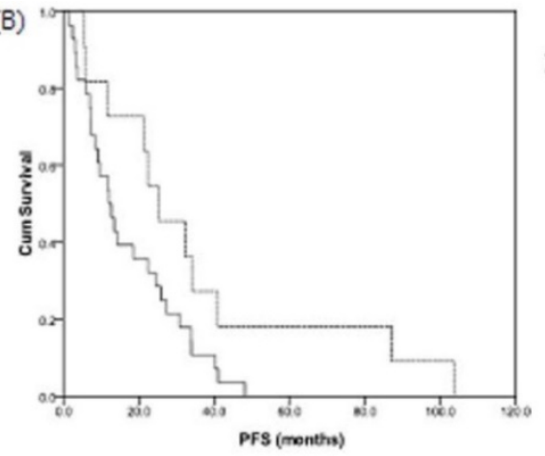

(C)

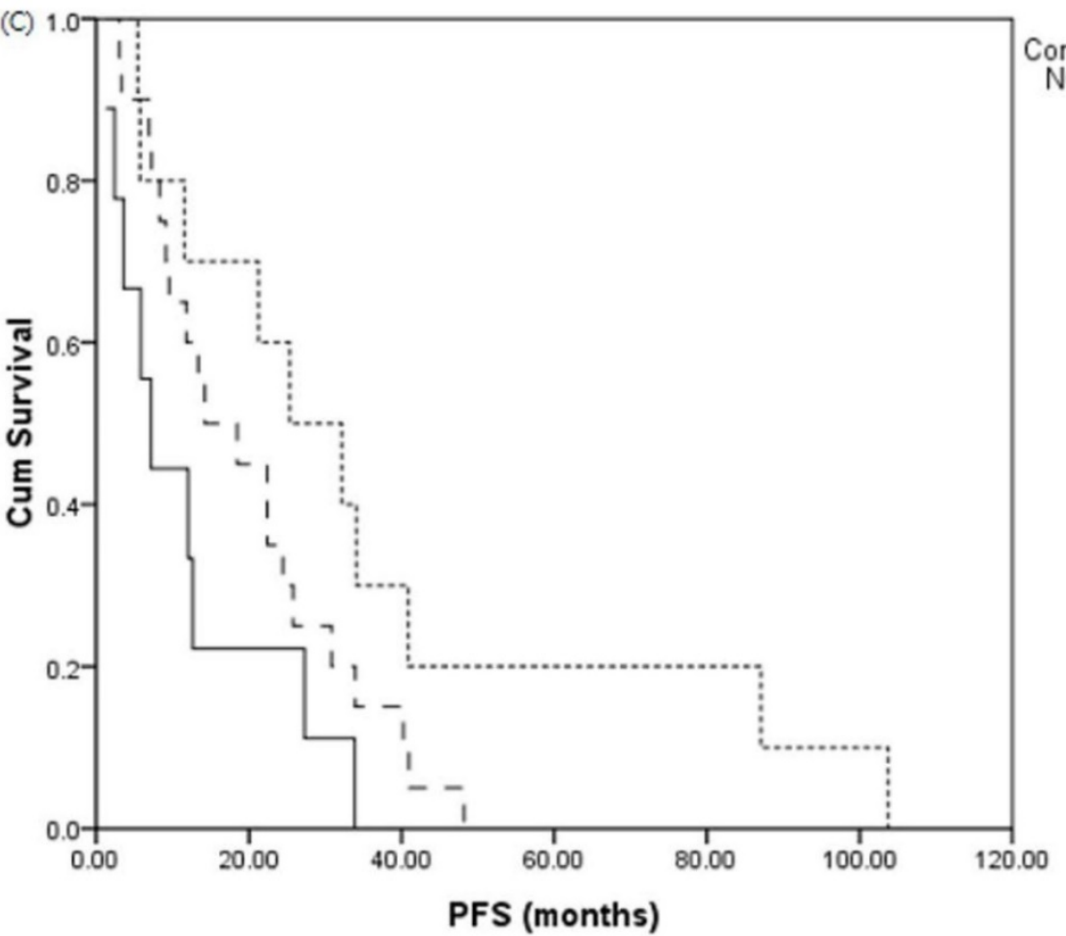

Combined NOTCH1 and NOTCH 3 expression

Both low

${ }^{-}$- Others

$\neg$ Both high

Figure 4. Expression of Notch1 (A) and Notch3 (B) effect disease-free survival (DFS) in lung adenocarcinoma. DFS analysis of patients with both low Notch1 and low Notch3 expression, both high Notch1 and high Notch3 expression in lung adenocarcinoma (C). Patients with adenocarcinoma who had both low Notchl and Notch3 expression $(n=23)$, both high Notchl and Notch3 expression $(n=14)$ and others $(n=38)$ in lung adenocarcinoma.

Notch1 and Notch3 have long been regarded as candidate molecules responsible for the development of lung cancer (14-19), while few studies have delineated a specific role for Notch2. Whether Notch2 has similar roles as Notch1 and Notch3 remains to be determined. Our results showed that Notch2 expression positively correlated with more advanced lung cancer stages and higher rate of cancer recurrence or metastasis. However, the expression of Notch2 had no impacts on DFS and OS in patients with lung adenocarcinoma. A recent study demonstrated that upregulation of Notch2 is a transcriptional aberration that contributes to preinvasive-to-invasive lung adenocarcinoma progression by inducing epithelial-mesenchymal transition (EMT) and nuclear atypia (21). These findings suggested that Notch2 plays a role during the early-stage lung adenocarcinoma progression. Recent work in Notch pathway activity in cancer cells shows EMT promotion (22-25) and enhancement of cancer stem cell characteristics (26), and interestingly, implicates Notch signaling in several cancers displaying resistance to conventional chemotherapy $(27,28)$. Higher activities of Notch pathway in lung cancer stem cells indicated worse survival of lung adenocarcinoma (29). Therefore, Notch pathway activity may encourage EMT formation and Notch2 is a key clue.

Several studies have examined the relationship between Notch expression and survival in patients with NSCLC. A recent meta-analysis indicated that higher expression of Notch1 by immunohisto- 
chemistry was associated with greater possibility of lymph node metastasis and more advanced TNM stages (30). Mimae et al. (21) revealed that paired upregulation of Notch2 and Six1 is associated with disease progression by clinicopathologic features and shorter DFS in patients with invasive adenocarcinoma. Our research showed that increased Notch2 expression was associated with more advanced lung cancer stages. Expression of Notch1 also positively correlated with advanced disease stages but the correlation was not statistically significant. In our study, the expression of Notch elements was assessed by RT-PCR, but patient selection bias and different laboratory techniques may cause distinct results. Therefore, further large-scale studies with consistent analytical methods are warranted.

Our study suggested that lung adenocarcinoma patients with both high expressions of Notch1 and Notch3 had poor DFS by Kaplan-Meier survival curve. Cox regression analysis showed that Notch3 expression remained the leading predictive factor of DFS. Yuan et al. (30) indicated that higher Notch3 expression was associated with poor overall survival rate of lung adenocarcinoma patients. Conversely, Liu et al. (31) demonstrated that higher Notch1, Notch2, JAG1, and DLL1 mRNA expression predicted better OS while elevated Notch3, JAG2, and DLL3 mRNA expression was associated with poor OS of lung adenocarcinoma patients by online database analysis. Though these findings have discrepant results, Notch 3 could be used as a potential marker to predict prognosis of lung adenocarcinoma. Since Notch signaling is initiated upon ligand binding to Notch receptor, a complete analysis including expressions of Notch and Notch ligands and their association with the prognosis of lung cancer should be explored further.

Though, the differential function between Notch1 to Notch4 is not well known. The activation status of Notch1 had poor prognostic impact on NSCLC (32) and was used to predict overall survival in the subgroup of p53-negative NSCLC patients (14, 33). Notch 1 also contributes to epidermal growth factor receptor (EGFR) tyrosine kinase inhibitor acquired resistance in NSCLC (34, 35). Besides, Notch3 expression is positively correlated with EGFR expression (36), and Notch3 overexpression associates with poor prognosis in NSCLC $(37,38)$. EGFR mutation status was not analysis at our study, but the mutation rate was relative higher in east Asia. Therefore, higher Notch 1 and Notch 3 expression was showed to relate to poor DFS of lung adenocarcinoma in our study.
In conclusion, patients with lung adenocarcinoma had higher Notch2 expression. Increased Notch2 expression was associated with more advanced lung cancer stages and higher cancer recurrence rate. Higher Notch1 and Notch3 expression were associated with poor prognosis in lung adenocarcinoma. Further investigations supporting clinical implementation of Notch signaling components are worthwhile.

\section{Acknowledgements}

The present study was supported by Research and Education Development Funds of Oncology, National Taiwan University Hospital, Yunlin Branch, Yunlin County, Taiwan.

We are indebted to Ms. Yi-hsiu Juan and Ms. Chao-Jung Chang for help with laboratory techniques, the First Common Laboratory of National Taiwan University Hospital, Yun-Lin Branch, for technical support.

\section{Competing Interests}

The authors have declared that no competing interest exists.

\section{References}

1. Siegel R, Ward E, Brawley O, et al. Cancer statistics, 2011: the impact of eliminating socioeconomic and racial disparities on premature cancer deaths. CA Cancer J Clin 2011;61:212-36.

2. Altekruse SF, Kosary CL, Krapcho M, et al. SEER Cancer Statistics Review, 1975-2007. Bethesda, MD: National Cancer Institute; 2007.

3. Osanyingbemi-Obidi J, Dobromilskaya I, Illei PB, et al. Notch signaling contributes to lung cancer clonogenic capacity in vitro but may be circumvented in tumorigenesis in vivo. Mol Cancer Res 2011;9:1746-54.

4. Miele L, Golde T, Osborne B. Notch Signaling in Cancer. Curr Mol Med 2006;6:905-18.

5. García Campelo MR, Alonso Curbera G, Aparicio Gallego G,et al. Stem cell and lung cancer development: blaming the Wnt, Hh and Notch signalling pathway. Clin Transl Oncol 2011;13:77-83.

6. $\mathrm{Xu} \mathrm{K}$, Moghal N, Egan SE. Notch signaling in lung development and disease. Adv Exp Med Biol 2012;727:89-98.

7. Galluzzo P, Bocchetta M. Notch signaling in lung cancer. Expert Rev Anticancer Ther 2011;11:533-40.

8. Collins BJ, Kleeberger W, Ball DW. Notch in lung development and lung cancer. Semin Cancer Biol 2004;14:357-64.

9. Carmeliet P. Mechanisms of angiogenesis and arteriogenesis. Nat Med 2000;6:389-95

10. Shawber CJ, Kitajewski J. Notch function in the vasculature: insights from zebrafish, mouse and man. Bio Essays 2004;26:225-34.

11. Gridley T. Notch signaling during vascular development. Proc Natl Acad Sci USA 2001;98:5377-8.

12. Westhoff B, Colaluca IN, D'Ario G, et al. Alterations of the Notch pathway in lung cancer. Proc Natl Acad Sci USA 2009;106:22293-8.

13. Baumgart A, Seidl S, Vlachou P, et al. ADAM17 regulates epidermal growth factor receptor expression through the activation of Notch1 in non-small cell lung cancer. Cancer Res 2010;70:5368-78.

14. Collins BJ, Kleeberger W, Ball DW. Notch in lung development and lung cancer. Semin Cancer Biol 2004;14:357-64.

15. Lin L, Mernaugh R, Yi F, et al. Targeting specific regions of the Notch3 ligand-binding domain induces apoptosis and inhibits tumor growth in lung cancer. Cancer Res 2010;70:632-8.

16. Wang NJ, Sanborn Z, Arnett KL, et al. Loss-of-function mutations in Notch receptors in cutaneous and lung squamous cell carcinoma. Proc Natl Acad Sci U S A 2011;108:17761-6

17. Konishi J, Kawaguchi KS, Vo H, et al. Gamma-secretase inhibitor prevents Notch3 activation and reduces proliferation in human lung cancers. Cancer Res 2007; 67: 8051-7.

18. Haruki N, Kawaguchi KS, Eichenberger S, et al. Dominant-negative Notch3 receptor inhibits mitogenactivated protein kinase pathway and the growth of human lung cancers. Cancer Res 2005;65:3555-61. 
19. Dang TP, Gazdar AF, Virmani AK, et al. Chromosome 19 translocation, overexpression of Notch3, and human lung cancer. J Natl Cancer Inst 2000;92:1355-7.

20. Seo JS, Ju YS, Lee WC, et al. The transcriptional landscape and mutational profile of lung adenocarcinoma. Genome Res 2012;22:2109-19.

21. Mimae T, Okada M, Hagiyama M, et al. Upregulation of notch2 and six1 is associated with progression of early-stage lung adenocarcinoma and a more aggressive phenotype at advanced stages. Clin Cancer Res 2012;18:945-55.

22. Yang Y, Ahn YH, Gibbons DL, et al. The Notch ligand Jagged2 promotes lung adenocarcinoma metastasis through a miR-200-dependent pathway in mice. J Clin Invest 2011;121:1373-85.

23. Talbot LJ, Bhattacharya SD, Kuo PC. Epithelial-mesenchymal transition, the tumor microenvironment, and metastatic behavior of epithelial malignancies. Int $\mathrm{J}$ Biochem Mol Biol 2012;3:117-36.

24. Wang Z, Li Y, Kong D, et al. The role of Notch signaling pathway in epithelial-mesenchymal transition (EMT) during development and tumor aggressiveness. Curr Drug Targets 2010;11:745-51.

25. Dasari V, Gallup M, Lemjabbar H, et al. Epithelial-mesenchymal transition in lung cancer: is tobacco the "smoking gun"?. Am J Respir Cell Mol Biol 2006;35:3-9.

26. Espinoza I, Pochampally R, Xing F, et al. Notch signaling: targeting cancer stem cells and epithelial-to-mesenchymal transition. Onco Targets Ther 2013;6:1249-59.

27. Wang Z, Li Y, Kong D, et al. Acquisition of epithelial-mesenchymal transition phenotype of gemcitabine-resistant pancreatic cancer cells is linked with activation of the notch signaling pathway. Cancer Res 2009;69:2400-7.

28. Wang Z, Li Y, Ahmad A, et al. Targeting Notch signaling pathway to overcome drug resistance for cancer therapy. Biochim Biophys Acta 2010;1806: 258-67.

29. Hassan KA, Wang L, Korkaya H, et al. Notch pathway activity identifies cells with cancer stem cell-like properties and correlates with worse survival in lung adenocarcinoma. Clin Cancer Res 2013;19:1972-80.

30. Yuan $\mathrm{X}, \mathrm{Wu} \mathrm{H}, \mathrm{Xu}$ H,et al. Meta-analysis reveals the correlation of Notch signaling with non-small cell lung cancer progression and prognosis. Sci Rep 2015;5:10338.

31. Liu ZY, Wu T, Li Q, et al. Notch Signaling Components: Diverging Prognostic Indicators in Lung Adenocarcinoma. Medicine (Baltimore) 2016;95:e3715.

32. Donnem T, Andersen S, Al-Shibli K, et al. Prognostic impact of Notch ligands and receptors in nonsmall cell lung cancer: coexpression of Notch-1 and vascular endothelial growth factor-A predicts poor survival. Cancer. 2010;116:5676-85.

33. Licciulli S, Avila JL, Hanlon L, et al. Notch1 is required for Kras-induced lung adenocarcinoma and controls tumor cell survival via p53. Cancer Res 2013;73:5974-84.

34. Xie $\mathrm{M}$, Zhang $\mathrm{L}$, $\mathrm{He} \mathrm{CS}$, et al Activation of Notch-1 enhances epithelial-mesenchymal transition in gefitinib-acquired resistant lung cancer cells. J Cell Biochem 2012;113:1501-13.

35. Xie M, He CS, Wei SH, et al. Notch-1 contributes to epidermal growth factor receptor tyrosine kinase inhibitor acquired resistance in non-small cell lung cancer in vitro and in vivo. Eur J Cancer 2013;49:3559-72.

36. Haruki N, Kawaguchi KS, Eichenberger S, et al. Dominant-negative Notch3 receptor inhibits mitogen-activated protein kinase pathway and the growth of human lung cancers. Cancer Res 2005;65:3555-61.

37. Ye YZ, Zhang ZH, Fan XY, et al. Notch3 overexpression associates with poor prognosis in human non-small-cell lung cancer. Med Oncol 2013;30:595.

38. Yi F, Amarasinghe B, Dang TP. Manic fringe inhibits tumor growth by suppressing Notch3 degradation in lung cancer. Am J Cancer Res 2013;3:490-9. 